\title{
Study of AC Losses in Superconducting Electrical Components for Electrical System Design
}

\author{
José-María Ceballos, Alfredo Alvarez, and Pilar Suarez \\ "Benito Mahedero" Group of Electrical Applications of Superconductors, \\ Industrial Engineering School, University of Extremadura \\ Apartado 382, Avenida de Elvas s/n \\ 06071 Badajoz, Spain \\ jmceba@unex.es, aalvarez@unex.es, psuarez@unex.es
}

\begin{abstract}
An experimental rig allowing us to perform AC loss measurements, on short $(10 \mathrm{~cm})$ tape samples of high-temperature superconductor $\mathrm{Bi}-2223 / \mathrm{Ag}$, was designed and tested.We propose a new set-up in order to determine AC losses in multilayer superconducting coils. We made a comparative AC losses study of an isolated tape and of the same tape in the same conditions except for the proximity of another tape, with and without current, located just over the first one. We present a serial of results obtains from different tapes, first we used multifilament BSCCO tape (1G) and YBCO tape (2G) with ferromagnetic substrate.
\end{abstract}

Keywords: AC losses, Superconductivity, Bi-2223 tapes, YBCO tapes, Superconducting coils.

\section{Introduction}

The purpose of the work was to study, characterize, and measure the different components of AC losses in superconductors that are part of such electrical systems as transformers, electrical motors, etc.

Firstly, we present a serial of experiments consisted in the evaluation of a single tape carrying current in a possible external magnetic field, obtained in collaboration with the Superconductor Physics Department within the Institute of Electrical Engineering from Slovak Academy of Sciences.

Finally, we focused in a tape as part of a multilayer coil, because this is the most usual way that the tape is used in electrical systems. The behavior of each section of tape is different from of an isolated piece because of the influence of the superconducting layer wound just next to it.

In order to analyze the different components of the AC loss including the influence of one section of tape by another wound together with it, we made a comparative study of an isolated tape and of the same tape in the same conditions except for the proximity of another tape, with and without current, located just over the first one. 


\section{Contribution to Technological Innovation}

The study of AC losses under the simultaneous action of the transport AC current and the external AC magnetic field is of prime importance for the reliable prediction of dissipation in electric power devices such as motors/generators, transformers and transmission cables. The reason for such a study is because, if the study of losses is an important part of the design of any electrical application, in superconducting electrical systems losses determine not only their efficiency but also the capacity of the corresponding cooling system.

\section{Experimental}

We carried out two different serial of experiments in order to determinate the $\mathrm{AC}$ losses in a sample of superconducting tape.

In the first one, we tried to find the contribution of the external magnetic field to the AC transport losses.

In the second one, we found the influence of the presence of nearby tapes on the AC losses in a single tape.

\subsection{Transport Losses in a Single Tape with External Magnetic Field}

For this experiment, the setup consists on an isolated tape feeding with a wide range of transport current and exposed, simultaneously to an external magnetic field (Fig. 1 shows a scheme of this configuration).

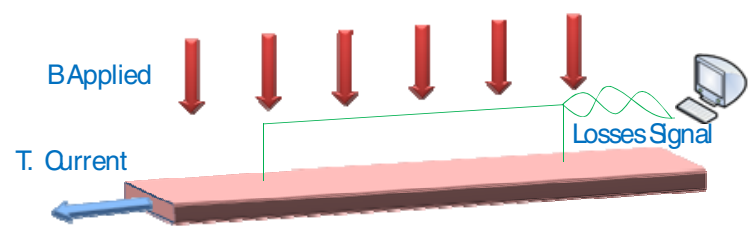

Fig. 1. Experimental arrangement for the measure of the transport losses in a single tape with external magnetic field

The main aim of this serial of experiment was to determine, by an electromagnetic method, the AC loss in a superconducting wire transporting the AC current $I_{T}=\sqrt{ } 2 I_{\text {rms }}$ $\cos (2 \pi f t)$ while exposed to the AC magnetic field $B_{e x t}=\sqrt{ } 2 B_{r m s} \cos (2 \pi f t+\varphi)$.

In particular, the dependence on the phase shift $\varphi$ between $\mathrm{I}_{\mathrm{T}}$ and $\mathrm{B}_{\mathrm{ext}}$ has been investigated.

To carry out this method, one has to resolve two main problems:

a) An independent supply of current into the sample and in the AC field magnet winding is necessary to keep the values of $\mathrm{I}_{\mathrm{rms}}$ and $\mathrm{B}_{\mathrm{rms}}$ constant while changing the phase difference. 
b) The distinction between the dissipation covered by the power supply for $\mathrm{I}_{\mathrm{T}}$ and the one delivered by the energizing system of the AC magnet is necessary to avoid a double count of dissipation in loss registration.

For measurement of transport loss the loop perpendicular to the wide face of the tape is used. AC loss per unit of length is determined by formula:

$$
P_{T}=I_{T} * V_{T} / L
$$

Where $I_{T}$ is the RMS value of transport current, $V_{T}$ is the part of RMS value of voltage from measuring loop which is in phase with transport current, and L is loop length. Perpendicular arrangement reduces the false voltage signal induced by external field. Fine compensation of the remaining false voltage is performed with the help of an adjustable mutual inductance linked to AC magnet current. Similar compensation is used in the case of the magnetization loss measurement, but linked now to the sample current. By this way the false signals were reduced satisfactory.

All the experiment with this set-up was implemented at frequency of $72 \mathrm{~Hz}$. The characteristics of the tape analyzed in this experiment are shown in table 1.

Table 1. Characteristics of the tape under test

\begin{tabular}{ll|ll}
\hline \hline \multicolumn{3}{l}{ High Strength American Superconductor } & \\
\hline Type & G & Width $(\mathbf{m m})$ & $4.2(+/-0.2 \mathrm{~mm})$ \\
Superconductor & $\mathrm{Bi}(\mathrm{Pb})-2223$ & Thickness $(\boldsymbol{\mu m})$ & $0.31(+/-0.02 \mathrm{~mm})$ \\
Fabrication Tech & PIT & $\mathbf{I}_{\mathbf{c}}(\mathbf{A})$ & $120 \mathrm{~A}$ \\
Matrix & Silver Alloy & Reinforcement & Stainless Steel \\
\hline \hline
\end{tabular}

\subsection{Transport Losses in a Single Tape under the Influence of Another Tape}

In many applications of superconducting tape in electrical devices, the tape must be wound in multilayer coils as in figure 2 . In such a case, every piece of tape is located very close to some other piece, in the next layer, along the coil. The proximity of these two parts of the circuit adds a new component (not necessarily positive) in the total loss of the multilayer coil that does not exist in the tape or single-layer coil loss. Therefore, we can divide the loss into 3 components:

a) The transport loss, $\mathrm{P}_{\mathrm{N}}$, which can be calculated by the Norris equation [1].

b) The magnetic loss, $\mathrm{P}_{\mathrm{mag}}$, due to the global magnetic field created by the complete coil.

c) The local loss, $\mathrm{P}_{\text {loc }}$, due to the proximity of turns in the same position of consecutive layers. So, the total loss, $\mathrm{P}_{\mathrm{T}}$, can be written as follows:

$$
\mathrm{P}_{\mathrm{T}}=\mathrm{P}_{\mathrm{N}}+\mathrm{P}_{\mathrm{mag}}+\mathrm{P}_{\text {loc }}
$$

To evaluate the new component of the loss, $\mathrm{P}_{\text {loc }}$, we designed and carried out the experiment described below.

Figure 3 shows the arrangement of the tapes for the measurement of the losses. In this case, the tape is not bent as in a coil, and therefore $\mathrm{P}_{\text {mag }}=0$ (no global magnetic field has to be taken into account). The electrical method is used to determine the 
losses in the longer tape through the measurement of the voltage between taps on the tape (see figure 3, circuits $C I$ and $C O$ ) or the emf in a contact-less loop (circuits $C L I$ and $C L O)$ [2].

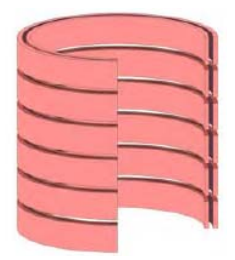

Fig. 2. Multi-layer coils carrying the same or different current in each layer. The proximity of the tapes in the same position of consecutive layers makes the AC loss different from in a single layer coil.

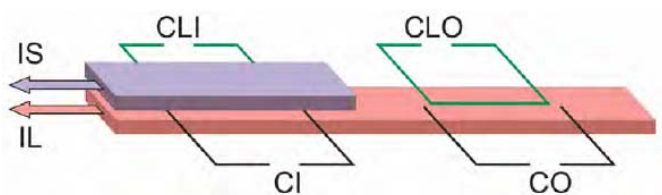

Fig. 3. Experimental arrangement of the tape (the longer) for the measurement of the losses both under and outside the influence of another tape (the shorter) very close to the former.

The shorter tape is located over the $C I$ and $C L I$ circuits, leaving the $C O$ a $C L O$ circuits outside its influence. The current $I_{L}$ in the longer tape and $I_{S}$ in the shorter one are independent but in phase for this study.

The measuring equipment picks up the waveforms of the currents through two Hall current probes, and the waveforms of the tap and loop voltages through four measurement amplifiers that filter and adapt the signals to be read by a data acquisition board (DAQ). All the waveforms have a whole number of periods (typically 5) at a frequency $f$, of $100 \mathrm{~Hz}$.

The characteristics of the different tapes under test are summarized in table 2.

Table 2. Characteristics of tapes under test

\begin{tabular}{lll}
\hline \hline & 1G Samples & 2G Samples \\
\hline Superconductor & Bi(Pb)-2223 & YBCO \\
Fabrication Tech & PIT & MOD/RABiTS \\
Matrix & Silver Alloy & ---- \\
Thickness $(\boldsymbol{\mu m})$ & $230 \pm 10$ & $150 \pm 20$ \\
Thickness $(\boldsymbol{\mu m})$ & $4.20 \pm 0.10$ & $4.40 \pm 0.15$ \\
$\mathbf{I}_{\mathbf{c}}(\mathbf{A})$ & 95 & 75 \\
\hline \hline
\end{tabular}

\section{Results and Discussion}

\subsection{Transport Losses in a Single Tape with External Magnetic Field}

In figure 4 , the dependence of transport loss $\mathrm{P}_{\mathrm{T}}$ as a function of the amplitude of the magnetic field is displayed. 


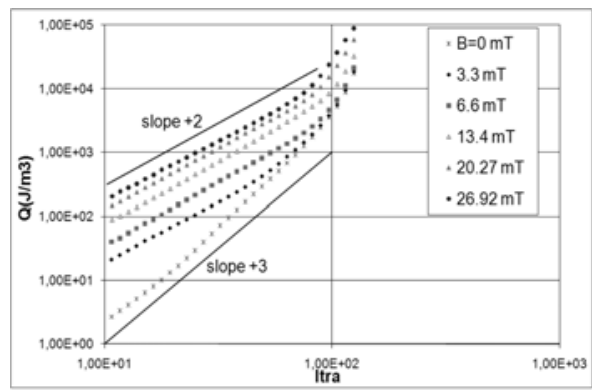

Fig. 4. Transport losses dependence with the amplitude of the perpendicular magnetic field (B) for different transport currents (Itra)

As we can see exits a strong dependence in the losses respect to the amplitude of the magnetic field.

The slope in the behavior of the transport losses change dramatically when a perpendicular magnetic field is applied.

We can also observe that this slope is even higher when the superconductor became to be saturated and the current starts to flow through the silver matrix [3].

In figure 5, the dependence of transport loss $P_{T}$ as a function of the phase shift between the transport current and magnetic field is displayed.

Although we initially supposed that the maximum of the losses occurs when the transport current and external field are in phase, as we can see in the figure 6 , it seems that the maximum of losses occurs in a range of shift angles between $30^{\circ}-50^{\circ}$, depending of the amplitude of the external magnetic field. The more the magnetic field amplitude increase, the more angle of the maximum of the losses, as we can see in the figure 6 , in which we present the variation of transport losses with fixed current (34A) under different field amplitudes. These transport losses have been normalized to the maximum.

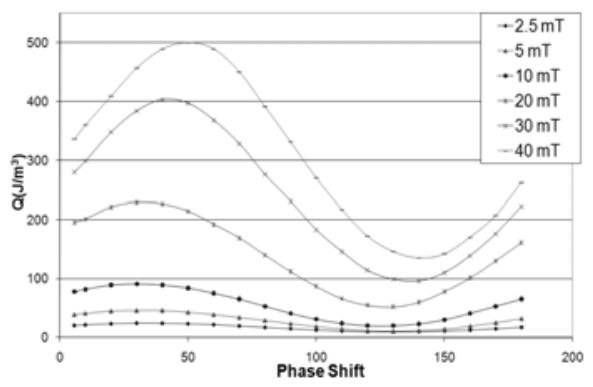

Fig. 5. Transport losses dependence with the phase shift in degrees, between transport current (34A) and magnetic field for different amplitudes of the magnetic field.

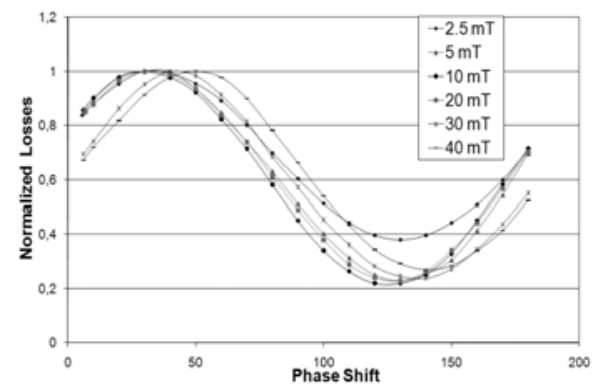

Fig. 6. Normalized to the maximum transport losses dependence with the phase shift between transport current (34A) and magnetic field for different amplitudes of the magnetic field 


\subsection{Transport Losses in a Single Tape under the Influence of Another Tape}

Figure 7 shows the loss in the $2 \mathrm{G}$ long tape, outside the influence of the shorter, as a function of the transport current $\mathrm{I}_{\mathrm{L}}$. The sample shows significantly higher losses compared with the theoretical values at currents lower than the critical current. This indicates that the losses in the $2 \mathrm{G}$ tapes are affected by the magnetic losses in the ferromagnetic substrates $[4,5-7]$.

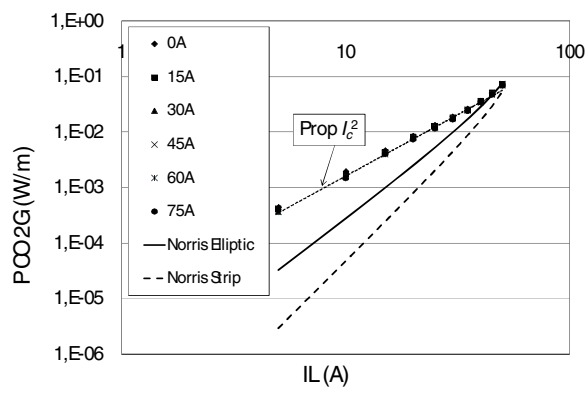

Fig. 7. Losses outside the influence of the short tape (probe $\mathrm{CO}$ ) for different currents $\mathrm{I}_{\mathrm{S}}$ (from 0 to $75 \mathrm{~A}$ ) in $2 \mathrm{G}$ sample. This loss corresponds to the transport loss in a $2 \mathrm{G}$ single tape at currents lower than critical current. It is been that the experimental measurements follow an $I_{L}^{2}$ dependence [8].

In Fig. 8 we have plotted the experimental data of losses in $2 \mathrm{G}$ sample and the sum with Norris's models with ferromagnetic losses. $F M 1$ corresponds to data from $[4,6]$ and $F M 2$ corresponds to data from [5, 6]. In general, the addition of the ferromagnetic losses $F M 1$ or $F M 2$ to the Norris Elliptical losses improves considerably the agreement with our experimental measurements as in [6]. However, our measurements fit to " $F M 2+$ Norris Elliptic" for all values of $\mathrm{I}_{\mathrm{L}}$ but only fit to " $F M 1+$ Norris Elliptic" for $\mathrm{I}_{\mathrm{L}}>25 \mathrm{~A}$.

In Fig. 9 a comparison of losses between $1 \mathrm{G}$ and $2 \mathrm{G}$ tapes for $\mathrm{I}<\mathrm{I}_{\mathrm{c}}$ is shown and we can see a significant difference between them but the most important one is the different slopes because of FM losses.

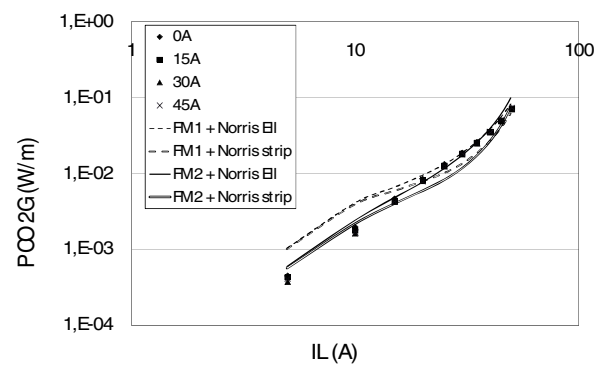

Fig. 8. Comparison between loss in $2 \mathrm{G}$ sample and theoretical curve making into account ferromagnetic losses in the substrate (FM) at values lower than the critical current

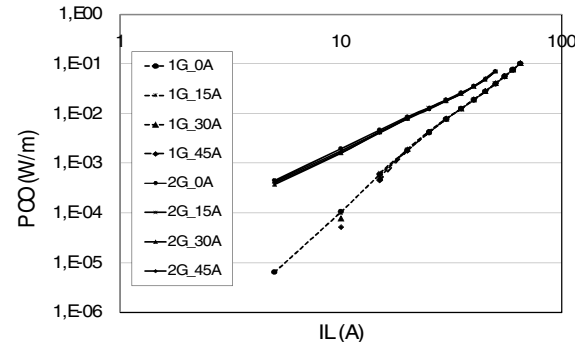

Fig. 9. Comparison between losses in $1 \mathrm{G}$ and $2 \mathrm{G}$ tapes measured through the probe $\mathrm{CO}$. These losses correspond to the transport losses in each single tape. 
The results for $1 \mathrm{G}$ setup show an expected behavior that is, the losses in probe $C I$ increase when $\mathrm{I}_{\mathrm{S}}$ increase concluding that the presence of consecutive superconducting layers affect to AC losses of the neighboring layers due to the dependence of the critical current with the transport current through the two tapes [9], but for $2 \mathrm{G}$ setup we can see in the figure 10 a different and interesting behavior. When $\mathrm{I}_{\mathrm{S}}$ increase below $I_{c}$ the losses in the long tape decrease but when $I_{S}$ increase above $I_{c}$ the losses in the long tape increase. Figure 11 shows a scheme of our arrangement to give a possible explanation of this effect.
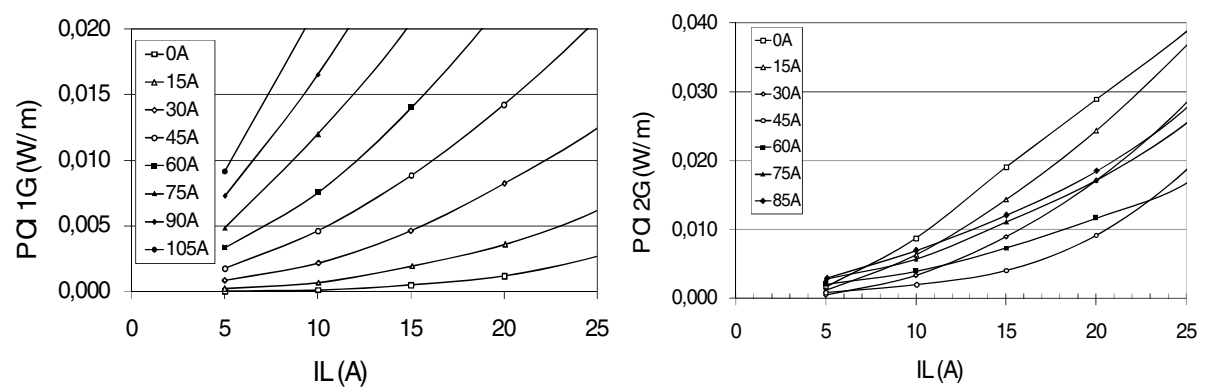

Fig. 10. Losses under the influence of the $1 \mathrm{G}$ and $2 \mathrm{G}$ short tape (probe $\mathrm{CI}$ ) for different currents IS (from 0 to $105 \mathrm{~A}$ in the $1 \mathrm{G}$ tape and from 0 to $85 \mathrm{~A}$ in the $2 \mathrm{G}$ tape)
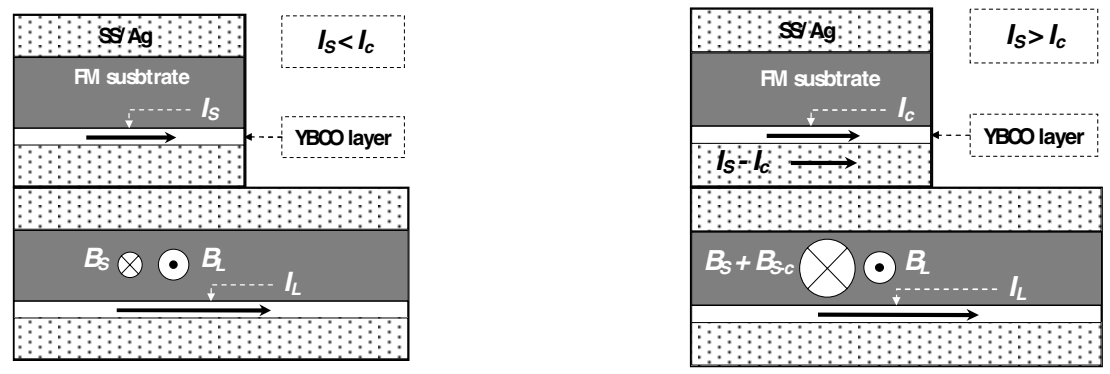

Fig. 11. Scheme of our arrangement. The right figure correspond to the case for $I_{S}<I_{c}$ and the left one is drawn the case for $I_{S}>I_{c}$. In both of them are the short and the long tapes. Spots represent Ag layers and stain steel covers, grey color represent FM substrates and white color represent YBCO layers. The layers are not at scale.

The short tape FM substrate and the conducting layers (Ag layer and stainless steel coverts) are located between short and long tapes YBCO layers. So when $\mathrm{I}_{\mathrm{S}}<\mathrm{I}_{\mathrm{c}}$, the magnetic field cause by $\mathrm{I}_{S}$ in the long tape FM substrate reduces the magnetic field in the same substrate due to $I_{L}$. This effect is stronger when $I_{S}$ increases producing a reduction of the losses in the long tape. However, when $I_{S}>I_{c}$, the current $\left(I_{S}-I_{c}\right)$ goes through the conducting layers increasing the magnetic field in the long tape FM substrate and the losses in the long tape [10]. 


\section{Conclusions}

An experimental set-up for the measurement of AC loss under the simultaneous action of the transport current and the magnetic field shifted in phase was developed and tested.

The results clearly show that the maximum loss is not at zero phase shift, and its position depends on the magnitude of the current and field.

Two similar arrangements for $1 \mathrm{G}$ and $2 \mathrm{G}$ assembled tapes have been constructed and studied in order to establish a comparison between them.

We have found a good agreement between our measurements and those estimated from bibliography for $2 \mathrm{G}$ single tape but it is necessary to carry on the study taking our own losses measurements in the ferromagnetic substrate.

Also we have shown a different behavior between $1 \mathrm{G}$ and $2 \mathrm{G}$ assembled tapes demonstrating that the existence and the location of ferromagnetic substrates are highly influent on the losses of the tapes. However, experiences with different $2 \mathrm{G}$ samples must be realized.

\section{References}

1. Norris, W.T.: Calculation of Hysteresis Losses on Hard Superconductors Carrying AC: Isolated Conductors and Edges of Thin Sheets. J. Phys. D, Appl. Phys. 3, 489-507 (1970)

2. Frolek, L., Soǔc, J., Laudis, A., Kovác, P., Husek, I.: Partitioning of Transport AC Loss in a Superconducting Tape into Magnetic and Resistive Components. IEEE Trans. Appl. Supercond. 11, 2967 (2001)

3. Gömöry, F., Janíková, E., Soǔc, J.: Resistive Losses in a High-Tc Wire Carrying AC Current Larger than Ic. Supercond. Sci. Technol. 15, 1345-1352 (2002)

4. Duckworth, R.C., Thompsom, J.R., Gouge, M.J., Lue, J.W., Ijaduola, A.O., Yu, D., Verebelyi, D.T.: Transport AC Losses Studies of YBCO Coated Conductors With Nickel Alloy Substrates. Supercond. Sci. Technol. 16, 1294-1298 (2003)

5. Duckworth, R.C., Gouge, M.J., Lue, J.W., Thieme, C.L.H., Verebelyi, D.T.: Substrate and Stabilization Effects on the Transport AC Losses in YBCO Coated Conductors. IEEE Trans. Appl. Supercond. 15, 1583-1586 (2005)

6. Gianni, L., Bindi, M., Fontana, F., Ginocchio, S., Martini, L., Perini, E., Zanella, S.: Transport AC Losses in YBCO Coated Conductors. IEEE Trans. Appl. Supercond. 16, 147-149 (2006)

7. Majoros, M., Ye, L., Velichko, A.V., Coombs, T.A., Sumption, M.D., Collings, E.W.: Transport AC Losses in YBCO Coated Conductors. Supercond. Sci. Technol. 20, 299-304 (2007)

8. Stravrev, S., Grilli, F., Dutoit, B., Ashworth, S.P.: Comparison of the AC Losses BSCCO and YBCO Conductors by Means of Numerical Analysis. Supercond. Sci. Technol. 18, 1300-1312 (2005)

9. Suarez, P., Alvarez, A., Perez, B., Ceballos, J.M.: Influence of the Current Through One Turn of a Multilayer Coil on the Nearest Turn in a Consecutive Layer. Journal of Physics, Conference Series 97, 012058 (2008)

10. Suarez, P., Alvarez, A., Ceballos, J.M., Perez, B.: Losses in 2G Tapes Wound Close Together: Comparison with Similar 1G Tape Configurations. IEEE Transactions on Applied Superconductivity 19, 2395-2398 (2009) 